Instituto Internacional de Investigación y Desarrollo Tecnológico Educativo INDTEC, C.A.

DOI: https://doi.org/10.29394/Scientific.issn.2542-2987.2019.4.14.16.328-346

OAI-PMH: http://www.indteca.com/ojs/index.php/Revista Scientific/oai

Artículo Original / Original Article

\title{
Práctica en las Líneas de Investigación y su Importancia para la Educación Universitaria
}

\author{
Autora: Yolanda del Carmen Díaz de Salas \\ Universidad Nacional Experimental "Rafael María Baralt", UNERMB \\ ydiazdesalas@yahoo.es \\ Trujillo, Venezuela \\ https://orcid.org/0000-0002-4785-2396
}

Resumen

Las Líneas de Investigación pertenecientes a la Universidad Nacional Experimental "Simón Rodríguez" (UNESR), Núcleo Valera, estado Trujillo, Venezuela corresponden a un área del conocimiento extensa, relacionada a un tema de interés, constituidas por el esfuerzo de un grupo de docentes, que se inclinan a investigar temas asociados, edificar conocimientos, tópicos conceptuales y hasta metodológicos. El propósito de este artículo, es interpretar las competencias investigativas del estudiante de educación avanzada en las Líneas de Investigación como espacios para la articulación, en torno a los ejes temáticos de los proyectos de indagación, individual o colectiva. Metodológicamente, la información se sustenta en el Paradigma Interpretativo, adoptando el Enfoque Cualitativo, con la aplicación de Entrevistas en Profundidad, dirigidas a cuatro (4) Informantes claves pertenecientes a cuatro Líneas de Investigación de la UNESR, Núcleo Valera. Dichas Líneas de Investigación reconocidas por las siguientes siglas: Fortalecimiento de la Educación Inicial (LinFEI); Gerencia y Tecnología (LinGERTEC); Centro de Investigación en Pobreza (CEDIPEPO); Lengua y Literatura y Arte (ILLA). Como resultado, se observó, que las Competencias Investigativas, surgen luego del proceso de comprensión, reflexión, intuición, creatividad adquirido en las Líneas de Investigación; Asimismo, de manera concluyente las evidencias, fueron la esencia del estudio y las actividades de las Líneas de Investigación donde deben posibilitar un trabajo cooperativo, basado en la episteme, articulado con los aspectos psicológicos, afectivos, culturales, científicos, éticos, ambientales y sostenibles en el tiempo.

Palabras clave: investigación; rendimiento de la educación; enseñanza superior.

Cómo citar este artículo:

Díaz de Salas, Y. (2019). Práctica en las Líneas de Investigación y su Importancia para la Educación Universitaria. Revista Scientific, 4(14), 328-346, e-ISSN: 2542-2987. Recuperado de: https://doi.org/10.29394/Scientific.issn.2542-2987.2019.4.14.16.328-346

Fecha de Recepción: 12-06-2019
Fecha de Aceptación: 23-09-2019
Fecha de Publicación: 05-11-2019 


\title{
Practice in Research Lines and its Importance for University Education
}

\begin{abstract}
The Research Lines belonging to the National Experimental University "Simón Rodríguez" (UNESR), Núcleo Valera, Trujillo, Venezuela state correspond to an area of extensive knowledge, related to a topic of interest, constituted by the effort of a group of teachers, who are inclined to investigate associated issues, build knowledge, conceptual and even methodological topics. The purpose of this article is to interpret the research competencies of the advanced education student in the Research Lines as spaces for articulation, around the thematic axes of research projects, individual or collective. Methodologically, the information is based on the Interpretive Paradigm, adopting the Qualitative Approach, with the application of Interviews in Depth, addressed to four (4) Key informants belonging to four Research Lines of the UNESR, Nucleus Valera. Said Research Lines recognized by the following acronyms: Strengthening Initial Education (LinFEI); Management and Technology (LinGERTEC); Poverty Research Center (CEDIPEPO); Language and Literature and Art (ILLA). As a result, it was observed that the Investigative Competences arise after the process of understanding, reflection, intuition, creativity acquired in the Research Lines; Likewise, the evidence conclusively was the essence of the study and the activities of the Research Lines where they should enable a cooperative work, based on the episteme, articulated with the psychological, affective, cultural, scientific, ethical, environmental and sustainable aspects in the time.
\end{abstract}

Keywords: research; educational output; higher education.

How to cite this article:
Díaz de Salas, Y. (2019). Practice in Research Lines and its Importance for University Education.
Revista Scientific, $4(14), \quad 328-346, \quad$ e-ISSN: $2542-2987 . \quad$ Recovered from:
https://doi.org/10.29394/Scientific.issn.2542-2987.2019.4.14.16.328-346

Date Received:

12-06-2019
Date Acceptance:

23-09-2019
Date Publication:

05-11-2019 


\section{Introducción}

Las Líneas de investigación pertenecen a la Universidad Nacional Experimental "Simón Rodríguez" (UNESR), permiten a los estudiantes egresados, adquirir habilidades, destrezas, conocimientos, para solucionar problemas de índole pedagógico, comunitario, personales y por ende, profesionales, desde una concepción científica e investigativa. Además, su formación y desarrollo se encuentra condicionado por los programas curriculares de las carreras a nivel universitario, guardando correspondencia con las políticas y demandas de la sociedad actual.

Respecto, al término de competencia en educación, Adla y Barraza (2011), argumentan:

El término competencia se ha vuelto una palabra omnipresente en el discurso educativo actual, sin embargo, su popularización académica no siempre ha sido acompañada de una teorización seria o responsable al respecto. Más allá de esta característica estructural del campo de estudio, que define y configura al término competencia, es posible observar dos indicadores que se entrelazan y nos hablan de su importancia para el pensamiento educativo contemporáneo: su multipresencia y su heurística (pág. 8).

Exponen estos autores, que la visión mostrada asume variados tipos inherentes a la educación, sin pretensiones de incumbirse en el sistema educacional. Con esto quiero decir, que en las competencias pensadas como modelo subyacen todos los elementos concordantes en la formación del individuo. Ante esta experiencia, de querer transformar la calidad en la enseñanza de la Educación Universitaria, urge innovar para que los actores implicados, sean capaces de participar y adquirir compromisos efectivos, en las políticas educativas actuales pensando en optimar el desarrollo de pueblos y conocer más a fondo los momentos históricos que ha vivido la humanidad.

Por ende, el caso educativo, que admite los procesos de investigación, donde se captan las creencias, conocimientos, valores y normas, tanto 
personales como grupales, circunda al investigador en pro de la realidad en su praxis investigativa. No obstante, juegan un papel predominante sus interacciones en grupos académicos, favorecedores de herramientas y elementos, que intervienen sobre el campo de conocimiento. Se observa entonces, la praxis educativa supeditada a las líneas de investigación, debido a la razón de ser de estas líneas donde se encierra el conocimiento, porque son considerados contextos de academia constantes, nutridos por las sapiencias, el intelecto y lo institucional.

Asimismo, para garantizar la educación, investigación y aprendizaje en las Universidades, se expone lo aludido por González, Zerpa, Gutiérrez y Pirela (2007):

En el ámbito educativo, cualquier profesional de la docencia vinculado al mundo de las instituciones, está llamado a desempeñar un papel clave como investigador de su propia práctica con la finalidad de mejorar su formación, su desempeño en el aula, escuela y comunidad en la búsqueda de un cambio organizacional hacia una transformación sociocultural (pág. 280).

Es decir, el rol de investigador, es misión fundamental de todo docente porque de él depende el incentivo que pueda ofrecer a los estudiantes para lograr aprendizajes óptimos en pro de la investigación y, por ende, en la formación de competencias investigativas. No obstante, si miramos la trayectoria histórica de la educación enfocada desde el progreso y motor de todo proceso desarrollista que involucre a la sociedad, donde se observe la vuelta de valores, que incentiven resguardar paralelismos culturales y ciudadanos se podría decir que constituyen el cimiento para formar y preparar recursos humanos, tan necesarios para una sociedad. Desde esta perspectiva, las instituciones educativas se convierten, en el lugar para la formación desde la adquisición, difusión de conocimientos relevantes, aunado al desarrollo de las capacidades intelectuales y productivas. 
En este sentido, se plantea la formación de profesionales, como eje central del desenvolvimiento universitario, en pro del desarrollo de un país, donde la educación sea reconocida como el pilar fundamental del desarrollo de éstos. Sin embargo, pese a este reconocimiento, la educación actual es cuestionada en sus resultados y procesos que lo conforman, sobre todo, la universitaria, a la cual se le reclama el seguir reproduciendo un desarrollo inadecuado en cuánto, a las competencias que deben alcanzar un docente frente a las expectativas y demandas de una sociedad cambiante.

Ahora bien, para ser competente, una manera de propiciarlo sería favorecer contextos que abarquen la investigación, entre estudiantes y profesores. Tomando en cuenta que, para ser un sujeto calificado de tener competencias, no significa tener que demostrar lo que conocemos, sino más bien diría tener la capacidad de hacer algo con lo que ya conocemos.

Asiduamente, como profesores queremos depositar en los receptores, en este caso los estudiantes un bagaje de conocimientos que han sido almacenados por la humanidad y luego, es fácil hacerles pruebas para evidenciar "si realmente conocen" lo que se les trasmitió, pero eso no transfiere ser competente. Por ende, toda competencia acarrea conocer, tener habilidad, actitud y claro está valores, lo que implica la dualidad del pensamiento y acción.

En ese orden de ideas, el proceso de investigar sea cual sea el enfoque que sirva de soporte, significa documentarse respecto a lecturas, estudio y análisis de escritos, además de reflexionar e interpretar lo cotidiano, que nos lleve a resolver adecuadamente, problemas del ámbito al cual pertenecemos y del contexto al que dependemos.

Considerando que, las estrategias podrían ser parte en el desarrollo de la praxis investigativa porque hacen adentrarse en un mundo mágico que es la indagación, pudiendo hacer entre otras cosas: crítico en la lectura de generalizaciones de investigaciones, que sean armónicas con igual temática 0 
de áreas afines, desde perspectivas de lectura, escritura o entelequia investigadora.

Algo semejante ocurre con estrategias, enfocadas en la revisión de proyectos de exploración, observar sus resultados preliminares con sentido crítico y de cientificidad, reflexionar sobre los propósitos que posea tal indagación, revisar ponencias, artículos, ensayos y conferencias publicadas en extensos, además de sinopsis de algunas notas acreditadas.

Por ello, una de las alternativas de solución ante estos síndromes está, el compartir la investigación en espacios académicos e investigativos, en grupo, en equipo que apoyen la reflexión, la exploración, en si todo lo que involucra en el aprendizaje relacionado a la investigación. El cual deben ser lugares constituidos en armonía, sustentados en la academia, el profesionalismo, la innovación y estar actualizados. Llegando a convertirse en comunidades de investigación, que se apropian de cultura, basada en diversas experiencias, lo que marca las diferencias en grupos académico.

Por consiguiente, estos grupos académicos son denominados Líneas de Investigación, sugiriendo por Chacín y Briceño (2001):

Al iniciar la planificación de una Línea de investigación, se hace necesario propiciar u nivel de discusión que permita establecer estrategias, parámetros 0 referentes que las identifiquen y caractericen en el contexto de su implantación. Así mimo, es imprescindible precisar el concepto con miras a organizar la investigación en las instituciones, justificando así la actividad que se realiza en las líneas en términos de su actividad y de los recursos en los que se apoya (pág. 46).

Por tanto, la razón de agruparse en Línea, es voluntario, con intereses temáticos comunes, que involucren profesores y estudiantes interesados en la investigación, capaces de insertarse en proyectos ofrecidos por la Línea de investigación. Sobre la base de estas ideas, el Sistema Educativo de Nivel Universitario cumple un rol esencial, al convertir a la propia educación en 
formación de factores precisos para el incremento de las competencias investigativas a favor, de darle solución a problemas y pretensiones que atribuyen las complejas decisiones del orden cultural, social y tecnológico.

Partiendo de esta aseveración y posicionados en la reflexión, mejorar la disposición de la educación universitaria, se ha convertido en un desafío que se debe tomar como un deber. Responsabilidad en la que indiscutiblemente, nos envolvemos todos, para poderlo llevar a la práctica en mejora de nuestra calidad de la educación, protagonizando en un futuro inmediato, la originalidad y la investigación. De ahí, la relevancia de la investigación donde se buscó interpretar las competencias investigativas del estudiante de educación avanzada en las Líneas de Investigación de la Universidad Nacional Experimental "Simón Rodríguez" (UNESR), Núcleo Valera.

\section{Marco Referencial}

El Marco Referencial creado en la presente investigación, efectúa un papel fundamental, porque permite, una clara y fácil comprensión del tema. En esta sección, se buscó información teórica, pertinente al estudio atendiendo la actitud de diferentes autores y fuentes bibliográficas, que sirven de concreción precisa, para definir aspectos esenciales tomados como posturas referentes a la investigación.

\subsection{Educación}

La concepción de la educación se impone por sí misma, como consecuencia de los cambios que se han manejado en la situación sociohistórica de la humanidad. La educación en este siglo XXI, debe proporcionar el aprendizaje de conocimientos teóricos y técnicos, adaptados a producción de conocimientos, ya que son las bases de la competencia del futuro.

En el Informe de la Organización de las Naciones Unidas para la Educación Científica y Cultural (UNESCO, 1998), enfatiza en los cuatro pilares 
de la educación, referidos a: "Aprender a Conocer, Aprender a Hacer, Aprender a Convivir y Aprender a Ser" (pág. 91). Estas bases, pueden dirigirse para dar oportunidad al estudiante y así, desenvolverse en la complejidad del mundo y a la vez, entenderlo debido a que se encuentra en permanente dinamismo, en lo concerniente al conocimiento y competencias que se pueden aplicar en los diferentes contextos: social, académico y comunitario.

De este planteamiento, es prioridad resaltar el Aprender a Conocer, como vía de desarrollo de herramientas o habilidades para la investigación, interpretar la situación próxima adquiriendo al mismo tiempo, las competencias para investigar. El Aprender a Hacer, está unido a la alineación profesional, aspecto curricular que amerita que las instituciones formadoras tomen conciencia que la educación es un servicio que se presta a la sociedad, por la necesidad de un profesional dotado de aspectos integrales, que pueda ejercer en su contexto laboral. De hecho, parte del aprender a conocer, mediante el conocer, el hombre se hace lucrativo a sus similares por la obra que realiza, y así solucionar la carencia del medio.

Dentro de la formación, se dibuja el Aprender a Vivir Juntos, visto desde la perspectiva de construir una plataforma social. Aprender a vivir juntos es educar, para las acciones envueltas en los valores de tolerancia, respeto, solidaridad, entre otros. En el Aprender a Ser, convergen los tres anteriores, es el esfuerzo por el desarrollo integral, armónico, donde emerja el equilibrio entre pensar, sentir y actuar.

\subsubsection{Educación Universitaria}

La Educación Universitaria, libera nuevas pautas para interrelacionarse estableciendo concordancia y tendencia de ausencia de los ambientes del desarrollo científico, caracterizados por la pérdida de condiciones de las universidades. En vista de esto, nosotros como seres humanos tenemos compromisos como profesionales, universitarios, intelectuales, con enormes e 
inevitables responsabilidades, no sólo de buscar el conocimiento, andar en la inquebrantable búsqueda de él, al tiempo de practicar nuestras profesiones de forma honesta, sino que tenemos el deber de establecer las nuevas circunstancias que nos brinden los ambientes, donde encinten abundantemente nuestros sueños.

De la misma forma, dialogar respecto a la universidad conduce a lo aludido por Tünnermann (2013), citado en García (2019), donde visualiza la universidad del futuro como: "...una universidad donde las ciencias, las humanidades y las artes encuentren un alero propicio; la innovación, la imaginación y la creatividad su morada natural" (pág. 248); de manera análoga, la universidad no solamente tiene el compromiso con profesionales y egresados sino ante servir a la comunidad, propiciando el desarrollo de una sociedad más justa y de confort donde el sujeto sea partícipe de una mejor calidad de vida para que pueda cumplir sus responsabilidades y deberes como ciudadano. Aunado a esto, su misión y visión deben estar dirigidas al desarrollo humano, donde todos sus actores sean parte de ella a la vez, que inserten avances culturales, científicos y de tecnología representantes actuales de la Educación Superior en nuestro país.

\subsection{Investigación}

La función Investigativa, se fundamenta en cuatro grandes temas: 1. los problemas centrales cuando se investiga; 2 . las concepciones fundamentales para describir los procesos de investigación; 3. el debate epistemológico; y 3. la estructura de los procesos de indagación. En lo que respecta, a los problemas actuales de la investigación en cualquier área, podemos afirmar que existen muchas nuevas propuestas, así como múltiples puntos de vista divergentes.

Los conceptos esenciales, permiten describir los procesos de investigación para diferenciarlos de otros distintos, parten de una serie de 
relaciones entre el conocimiento y sus características, la acción, investigación y el individuo; relaciones que explica Padrón (1999), afirmando que: "la investigación es un proceso típico y específico de producción de conocimiento sistemático-socializado, orientado a apoyar el éxito de las acciones educativas" (pág. 33).

En lo que se refiere, al debate epistemológico, postula que los enfoques epistémicos se pueden agrupar sobre la base de dos criterios: la concepción del conocimiento y la idea de métodos válidos para producirlo, criterios que permiten distinguir tres enfoques: 1. Empirista-inductivo; 2. Racionalistadeductivo; y 3. El fenomenológico-introspectivo. Todos los tres, constituyen la base de la investigación disciplinaria, pluridisciplinaria e interdisciplinaria y se complementan en la transdisciplinariedad.

Por ende, los estilos de pensamiento del investigador, reflejan una estrecha relación con los enfoques epistemológicos mencionados, porque van a incidir en las perspectivas sobre el proceso investigativo, donde se evidencian por medio de las creencias personales, el abordaje social y su visión de la realidad, en su práctica investigativa.

\subsection{Competencias Investigativas}

Referirse al término de competencias, alude concebir el conjunto de conocimientos, destrezas y aptitudes del sujeto para enfrentar los problemas de cualquier índole. Al respecto, Zabala y Laia (2008), citados por TrujilloSegoviano (2014):

El desarrollo por competencias en las universidades son una alternativa a la formación conceptual, centrado y reducido a unos aprendizajes de unos conocimientos, por encima de las habilidades para el desarrollo de la profesión... las pruebas y los criterios de evaluación de la mayoría de los exámenes y oposición propician el carácter disociado entre teoría y práctica, ya que el alumno memoriza el temario, con el fin de desarrollar los conocimientos adquiridos en una prueba, y no para poder 
aplicarlos (pág. 310).

Al respecto, los autores suponen, formar en competencias, es un proceso articulado, entre la triada del conocer y el hacer, para llegar al ser donde el individuo esté apto para actuar, basándose en sus potencialidades logradas, lo cual garantizaría el éxito en su actuar, con ética, conocimiento y un excelente hacer, relacionado por el conocer y el ser.

Respecto, a las competencias investigativas se corresponden con las habilidades, conocimiento, actitud y experiencia que debe poseer el investigador cuando desea realizar una indagación. Además, alcanzar la competencia investigadora significa tener capacidad de corregir problemas en el proceso de investigación sobre la base de la variabilidad y elasticidad metodológica, conociendo posibilidades y restricciones de cada estrategia metodológica, que se crea pertinente desarrollar.

Por ende, para entender el término investigación, debemos estar conscientes de la magnitud de su relevancia en cuanto, a descubrir otras formas de conocer, llenas de curiosidad, es decir, se debe descubrir lo que se desconoce, para sobre esto ir indagando acerca de problemas que aún, omitimos en ciertos momentos, por no poseer un lugar privilegiado en nuestras mentes en lo que a investigación se refiere.

Para fomentar las competencias investigativas en los estudiantes de la Educación Superior, es recomendable ofrecerles recursos para que puedan crear, planear y diseñar proyectos dirigidos a la indagación donde sean capaces de acuerdo a sus resultados, poder orientarse para tomar decisiones acertadas, a favor de solucionar problemáticas inherentes e inmediatas, además se deben construir teorías con originalidad para realimentar su praxis como profesional dirigidas al bien social. 


\subsection{Líneas de Investigación}

En términos generales, corresponden a un área del conocimiento generalmente, extensa relacionada a un tema de interés. Están constituidas por el esfuerzo de un grupo de docentes, que se inclinan a investigar en temas asociados, construir conocimientos, por inclinación disciplinaria, temática, conceptual y hasta metodológica, la validación de las mismas, es decisión de las autoridades de las universidades.

En el caso, de las líneas de investigación de la Universidad Nacional Experimental "Simón Rodríguez" (UNESR, 2011), éstas se rigen por el Reglamento General de Educación Avanzada, en el que se expone en su Artículo 100: "las líneas de investigación adscritas a programas de investigación serán instancias operativas garantes de la realización de diversas fases de los proyectos de investigación, que deben cursar los participantes como unidades curriculares obligatorias de los respectivos planes de estudio..." (pág. 29). Con esto quiero decir que, los maestrantes deben asistir a las líneas con obligatoriedad y allí, ir construyendo su Trabajo de Grado el cual se desarrolla con una investigación educativa.

\section{Metodología}

\subsection{Paradigma Epistemológico de la Investigación}

Toda indagación, responde filosóficamente según, Pasek y Matos (2006): "pues postulan variadas opciones con implicaciones ontológicas, epistemológicas, axiológicas y metodológicas diferentes" (pág. 119).

Ante el planteamiento ontológico, la concepción del hombre para el estudio, es considerado como el ser que construye y reconstruye en su interacción social, cuya reconstrucción se da desde lo exterior a lo interior. Por su parte, la relación sujeto-objeto en el conocimiento (epistemológica) está centrada en que el conocimiento y la subjetividad surgen de y en la interpretación y descripción de los fenómenos entre los sujetos de estudio. De 
allí, el propósito de develar, predecir, interpretar y dar significado a la vida de las unidades sociales.

Es así como la ontología y epistemología depende la metodología, la cual permitió llegar a la construcción y reconstrucción de significado a partir de los actores con la aplicación de métodos y técnicas basados en procesos hermenéuticos, diálogos que suceden intentando sacar sentido de sus expresiones desde la interpretación de los significados que tiene para las personas implicadas.

Por lo antes expuesto, se consideró el enfoque cualitativo como la vía que orienta este trabajo, por cuanto permite comprender la completa y subjetiva realidad de los actores educativos. Así, a la luz de este enfoque, donde el objeto de estudio es interpretar las competencias en el campo de la investigación del estudiante de educación avanzada en las Líneas de Investigación de la UNESR, Núcleo Valera.

\subsection{Método: Fenomenológico}

El enfoque cualitativo, bajo el método fenomenológico, se origina en la antropología que para Goetz y LeCompte (1988): "consiste en una teoría de la descripción; concepción que reafirma la percepción de que la fenomenología es el reflejo de la realidad, producto de la interacción humana" (pág. 115). Para reafirmar lo expuesto por los citados autores, se considera en forma analítica, que la investigación fenomenológica es interpretativa, con valor al protagonismo humano para crear interpretaciones significativas, en el entorno social y físico; por ende, las interacciones humanas deben ser valoradas en su magnitud, porque de ello depende la dimensión de la realidad.

De acuerdo con lo antes expuesto, los actores clave (egresados de la Maestría Ciencias de la Educación de la UNESR), aportaron una serie de cualidades en forma de descripciones completas de acontecimientos, interacciones y actividades que llevaron a la autora, al descubrimiento de 
categorías y relaciones que ayudaron a la interpretación de dichos datos.

\subsection{Recorrido Metodológico}

De manera que, el proceso o recorrido metodológico utilizado por la investigadora para producciones inductivas, analítica, en pro de la generación de teoría que caracteriza a los estudios doctorales se fundamentó en el recorrido metodológico planteado por Rodríguez, Gil y García (1996), son: "cuatro fases fundamentales en el proceso de investigación cualitativa: Preparatoria, Trabajo de Campo, Analítica, Informativa" (pág. 3). Estos procesos son originados por la relación entre componentes de una realidad social, presentada en este estudio en categorías emergentes. Luego, se constituyeron en una construcción dialéctica que generó un producto intelectual, basado en la comprensión de su totalidad.

\section{Análisis e interpretación de los resultados}

Los resultados obtenidos, se abordaron aplicando Entrevistas en Profundidad, realizada a cuatro Informantes Clave pertenecientes a cuatro Líneas de Investigación de la UNESR, Núcleo Valera. Dichas Líneas de Investigación reconocidas por las siguientes siglas: Fortalecimiento de la Educación Inicial (LinFEI); Gerencia y Tecnología (LinGERTEC); Centro de Investigación en Pobreza (CEDIPEPO); Lengua y Literatura y Arte (ILLA). Todas las entrevistas a las cuatro (4) maestrantes se grabaron con una duración de aproximadamente (20) veinte minutos, en otras (30) treinta minutos, todo dependiendo de la espontaneidad de cada uno de ellas. Dicha interactividad con los maestrantes egresados, se mantuvo en un clima de confianza y ameno.

Una vez finalizada cada una de las entrevistas, la investigadora procedió a la transcripción de la conversación, tal y como se llevó a efecto, para luego someterlas a la interpretación. Por consiguiente, para lograr el 
resultado de las categorías halladas la autora hizo una clasificación de las expresiones de las informantes claves, de manera de lograr la extracción de competencias.

Respecto, a, los hallazgos generados surgen: la Categoría de Competencias Afectivas "Saber Ser", está inmersa en el cuarto pilar de la educación, observando que el acto educativo debe ayudar al desarrollo integral de cada persona. Seguidamente, la autora obtiene la segunda categoría denominada Categoría de Competencias Cognoscitivas, "Saber Conocer" relacionadas al conjunto de conocimientos y a sus procesos de desarrollo. Por último, la Categoría de Competencias Procedimentales "Saber Hacer" la cual se enfocó en este estudio, en habilidades, destrezas y procedimientos.

En conclusión, se pudo develar que las actividades realizadas en las Líneas de Investigación de la UNESR, posibilitan un trabajo de investigación, que permite una construcción individual y colectiva de conocimientos. A continuación, se presenta la figura 1, que refleja los hallazgos y el proceso de Triangulación, desde la óptica de la autora.

Figura 1. Representación Gráfica de los Hallazgos y el Proceso de Triangulación, desde la Óptica de la Autora.

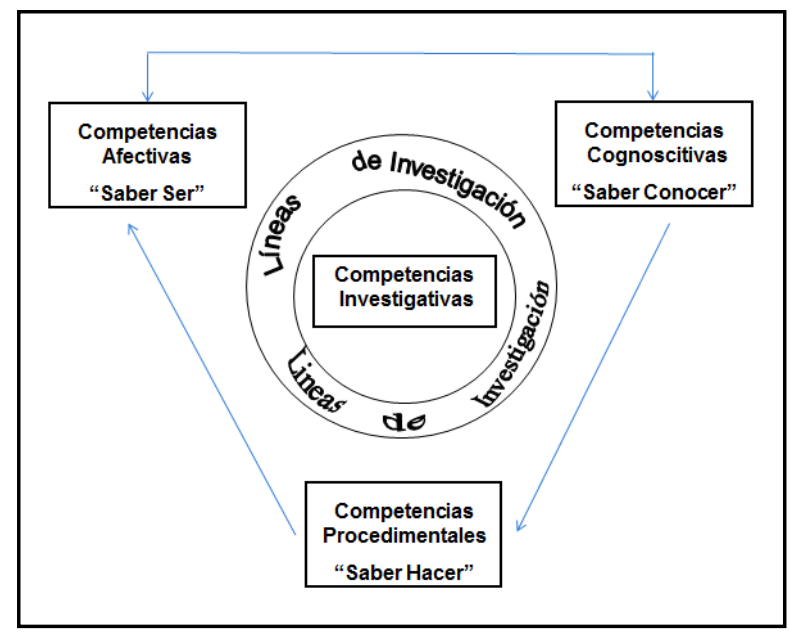

Fuente: La Autora (2018). 


\section{Consideraciones Finales}

Las consideraciones finales que sintetizo, van de acuerdo con el objetivo del artículo y la temática tratada: Las Competencias Investigativas, surgen luego de un proceso de comprensión, reflexión, intuición, creatividad que se adquieren o desarrollan en las Líneas de Investigación, se entiende que no es solamente la temática cognoscitiva, son otros temas culturales que integran o complementan las competencias para aprender a investigar.

A través, de las interrogantes efectuadas por la investigadora, en las Entrevistas en Profundidad las evidencias fueron, la esencia del estudio y las respuestas acordes, que se convirtieron en el brillo para interpretar y llegar a consideraciones propias del contexto de estudio. Además, de la colaboración y participación de las egresadas de Maestría en Ciencias de la Educación de la UNESR, que también asistieron a las Líneas de Investigación para adquirir los conocimientos en Competencias Investigativas.

En el ámbito de las líneas de investigación, en su cotidianidad van conociendo cada vez que comparten actividades grupales, en equipo en situaciones de riesgo, de dificultades. En tal sentido, es necesario que en las líneas de investigación de hoy día, se pueda proveer de actividades que permitan el fomento de las relaciones interpersonales óptimas, que permitan el trato social cotidiano, donde se establezcan reglas y normas que cumplir en pro de dicho desarrollo de las competencias investigativas.

Fue muy enriquecedor llegar a teorizar, como producto del pensamiento creativo, ingenio, pensar de manera divergente, donde expresé mi subjetividad basada en los actores claves, pues entendí la intersubjetividad. Es elogioso, el rol de integrantes de Líneas de Investigación, profesores, estudiantes, que se rigen por un plan, que al ejecutarlo aparecen aspectos no precisados en el papel, como es la parte afectiva. 


\section{Referencias}

Adla, J., \& Barraza, J. (2011). Competencias y Educación. Miradas múltiples de una Relación. Primera Edición, ISBN: 978-607-9003-012. México: Instituto Universitario Anglo Español, A.C.; Red Durango de Investigadores Educativos, A.C. Recuperado de:

https://redie.mx/librosyrevistas/libros/competencias y educacion.pdf

Chacín, M., \& Briceño, M. (2001). Cómo Generar Líneas de Investigación: Sugerencias Prácticas para Profesionales y Estudiantes. Segunda Edición, ISBN: 980-6230-04-3. Caracas, Venezuela: Publicaciones del Decanato de Postgrado de la Universidad Nacional Experimental Simón Rodríguez. Recuperado de:

http://www.quadernsdigitals.net/index.php?accionMenu=hemeroteca.D escargaArticulolU.descarga\&tipo=PDF\&articulo id $=10187$

García, S. (2019). La Gerencia de las Direcciones de Cultura y la Responsabilidad Social Universitaria desde una Cosmovisión Integrativa. Revista Scientific, 4(11), 239-259, e-ISSN: 2542-2987. Recuperado de:

https://doi.org/10.29394/Scientific.issn.2542-2987.2019.4.11.12.239$\underline{259}$

Goetz, J., \& LeCompte, M. (1988). Etnografía y Diseño Cualitativo en Investigación Educativa. ISBN 10: 847-1123-20-7; ISBN 13: 978-847112-320-6. Madrid, España: Editorial Morata.

González, N., Zerpa, M., Gutiérrez, D., \& Pirela, C. (2007). La investigación educativa en el hacer docente. Laurus, 13(23), 279-309, e-ISSN: 1315-883X. Recuperado de:

http://www.redalyc.org/articulo.oa?id=76102315

Padrón, J. (1999). La estructura de los Procesos de Investigación. Revista de Educación y Ciencias Humanas, Año VII(15), 33-48, ISSN: 13151762. Venezuela: Decanato de Postgrado de la Universidad Nacional 
Experimental Simón Rodríguez.

Pasek, E., \& Matos, Y. (2006). Cinco paradigmas para abordar lo real. Telos, 8(1), 106-121, e-ISSN: 1317-0570. Recuperado de:

http://www.redalyc.org/articulo.oa?id=99318655008

Rodríguez, G., Gil, J., \& García, E. (1996). Metodología de la Investigación

Cualitativa. Granada, España: Ediciones Aljibe. Recuperado de: https://cesaraguilar.weebly.com/uploads/2/7/7/5/2775690/rodriguez gil 01.pdf

Trujillo-Segoviano, J. (2014) El enfoque en competencias y la mejora de la educación. Ra Ximhai, 10(5), 307-322, e-ISSN: 1665-0441. Recuperado de: http://www.redalyc.org/articulo.oa?id=46132134026

Tünnermann, C. (2013). Diez respuestas de la educación superior a los desafíos contemporáneos. Universidades, (56), 5-14, ISSN: 00418935. Recuperado de:

http://www.redalyc.org/articulo.oa?id=37331245002

UNESCO (1998). Declaración Mundial sobre la Educación Superior en el siglo XXI: Visión y Acción. Conferencia Mundial sobre la Educación Superior en el siglo XXI: Visión y acción, Código del documento: ED.98/CONF.202/3. París, Francia: Organización de las Naciones Unidas para la Educación, la Ciencia y la Cultura. Recuperado de: http://www.unesco.org/education/educprog/wche/declaration spa.htm

UNESR (2011). Reglamento General de Educación Avanzada de la Universidad Nacional Experimental Simón Rodríguez. Aprobado por el Consejo Directivo en la sesión no 473, de fecha 26 de julio. Venezuela: Decanato de Postgrado de la Universidad Nacional Experimental Simón Rodríguez. Recuperado de:

http://www.espadultos.org.ve/doc/REGLAMENTO.pdf

Zabala, A., \& Laia, A. (2008). 11 ideas clave: Cómo aprender y enseñar competencias. ISBN: 978-84-7827-500-7. España: Editorial Graó. 


\section{Yolanda del Carmen Díaz de Salas \\ e-mail: ydiazdesalas@yahoo.es}

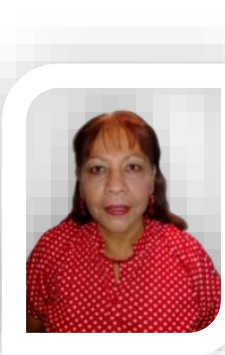

Nacida en la ciudad de Valera, estado Trujillo, Venezuela, el 21 de noviembre del 1956. Profesional universitaria en el área de Gerencia de Recursos Humanos; Doctora en Ciencia de la Educación, egresada de la Universidad Nacional Experimental "Rafael María Baralt" (UNERMB); $\mathrm{He}$ participado como Docente Contratada en la Universidad Nacional Experimental Politécnica de la Fuerza Armada (UNEFA), Betijoque, estado Trujillo; Desempeño el cargo de docente de aula, en la Universidad Bolivariana de Venezuela (UBV), Municipio Valera, estado Trujillo. Además, poseo conocimientos avanzados en cuanto al manejo de Windows XP; Microsoft Office (Word, Excel, PowerPoint); Lulo Software; AutoCAD Avanzado 2D e Internet.

El contenido de este manuscrito se difunde bajo una Licencia de Creative Commons ReconocimientoNoComercial-Compartirlgual 4.0 Internacional 